\title{
DECOLORACIÓN DEL ANARAJADO DE METILO EMPLEANDO EL SISTEMA FENTON
}

\author{
Carlos Gamarra Güere ${ }^{\mathrm{a}}$, Adolfo La Rosa-Toro Gómez ${ }^{\mathrm{b}}$
}

\section{RESUMEN}

En el Perú sólo se realiza el tratamiento del 29,1\% de las aguas residuales domésticas urbanas. Este tratamiento se realiza en 143 plantas, vertiéndose el resto a los ríos, lagos, y al mar, por lo que se hace necesario darle más importancia a la gestión de recursos hídricos. La industria textil, que emplea grandes cantidades de pigmentos para el teñido no trata adecuadamente sus efluentes siendo parte del problema. El proceso Fenton es una alternativa para el tratamiento de los efluentes del proceso de teñido, siendo el contaminante más importante los compuestos azoicos. El proceso Fenton ofrece la posibilidad de reusar y/o reciclar las aguas con una alta eficiencia $(86 \%)$ en pocos minutos.

Palabras clave: Fenton, decoloración, oxidación, remediación ambiental

\section{DISCOLORATION OF METHYL ORANGE BY FENTON PROCESS}

\begin{abstract}
In Peru only treat $29.1 \%$ of urban domestic wastewater is performed. This treatment is carried out at 143 industries, pouring the rest into rivers, lakes and the sea, so it is necessary to give more importance to the management of water resources. The textile industry, which uses large amounts of dye pigments not properly treat their effluents, being part of the problem. The Fenton process is an alternative for the treatment of effluents from the dyeing process, the most important pollutant azo compounds. The Fenton process provides the ability to reuse and / or recycle water with high efficiency $(86 \%)$ in a few minutes.
\end{abstract}

Keywords: Fenton, discoloration, oxidation, environmental remediation

\section{INTRODUCCIÓN}

La combinación de sales ferrosas solubles y peróxido de hidrógeno en proporciones adecuadas se denomina reactivo de Fenton (Ec.1); si el ion ferroso se sustituye por el hierro (III) se denomina reactivo Fenton-like (Ec.2). La regeneración de hierro ferroso ocurre en reacciones del fierro (III) con los radicales intermedios del proceso de degradación de los radicales hidroxilo, las cuales están representadas por las Ec. 3 y 4, todas estas ecuaciones ya fueron estudiados y publicados en la literatura ${ }^{1-3}$.

a Licenciado en Química, Laboratorio de Electroquímica Aplicada Facultad de Ciencias, Universidad Nacional de Ingeniería. e-mail: carlosgamarra@iqsc.usp.br

$b^{*}$ Profesor Principal y Jefe del Laboratorio de Investigación en Electroquímica Aplicada de la Facultad de Ciencias, Universidad Nacional de Ingeniería. Av. Túpac Amaru 210, Rímac e-mail: toro@uni.edu.pe 


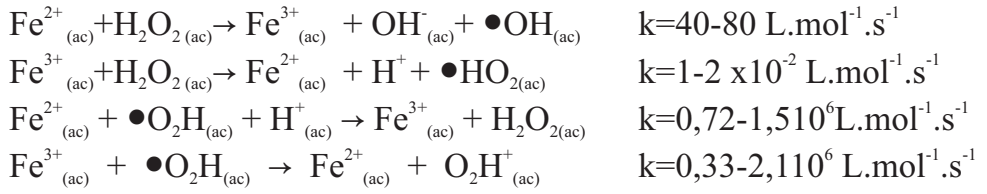

El anaranjado de metilo (AM) es un colorante de tipo azoico que es utilizado como patrón para el estudio de oxidación (decoloración) de efluentes coloreados de la industria textil. La estructura del AM se muestra en la figura 1, el cual posee el grupo cromóforo (color de la sustancia $)^{4}$, en ella se observa que el doble enlace del grupo funcional azo cambia a enlace simple al disminuir el $\mathrm{pH}$ del medio facilitando el rompimiento del enlace $-\mathrm{N}=\mathrm{N}$ - mediante las sustancias oxidantes generados en el proceso Fenton consiguiendo el objetivo de decolorar el agua.
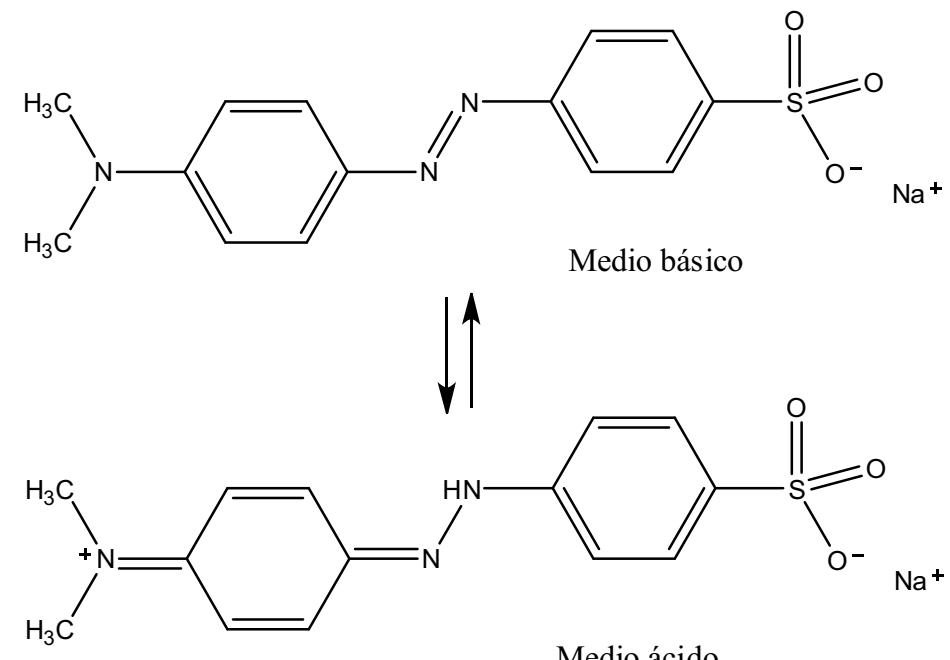

Figura 1. Estructura del Anaranjado de metilo en medio básico y en medio ácido

Los radicales hidroxilos generados en el proceso Fenton posibilitan la oxidación de la molécula orgánica rompiendo el enlace del grupo azo; sin embargo la mineralización completa de las moléculas orgánicas es posible mediante la reacción mostrada en la Ec.5:

$$
\bullet \mathrm{OH}+\mathrm{C} \rightarrow \mathrm{CO}_{2}+\mathrm{H}_{2} \mathrm{O}
$$

Sin embargo, una desventaja del proceso de Fenton es que no es capaz de mineralizar por completo todos los compuestos orgánicos presentes en el agua. Esto se debe a que las especies generadas en las últimas etapas de la oxidación (ácidos carboxílicos y dicarboxílicos) forman complejos estables con el hierro, evitando así el ataque de los radicales $\bullet \mathrm{OH}$. Deteniendo el ciclo catalítico antes de alcanzar la mineralización completa ${ }^{5,6}$ 


\section{Degradación del anaranjado de metilo (AM)}

Brillas $^{2}$ y Guivarch ${ }^{7}$ plantearon el mecanismo de decoloración del AM como una parte de las reacciones asociadas al descenso del DQO y de la mineralización de todo el compuesto orgánico a $\mathrm{CO}_{2}$. En la figura 2 se muestra la propuesta del mecanismo de reacción en la cual se observa que la molécula de AM inicia la reacción de oxidación con los radicales hidroxilo producidos por el proceso Fenton (Ec.1) y prosigue generando intermediarios orgánicos rompiendo los anillos aromáticos hasta su mineralización completa formando $\mathrm{CO}_{2} \mathrm{y}_{2} \mathrm{O}$ como productos terminales.

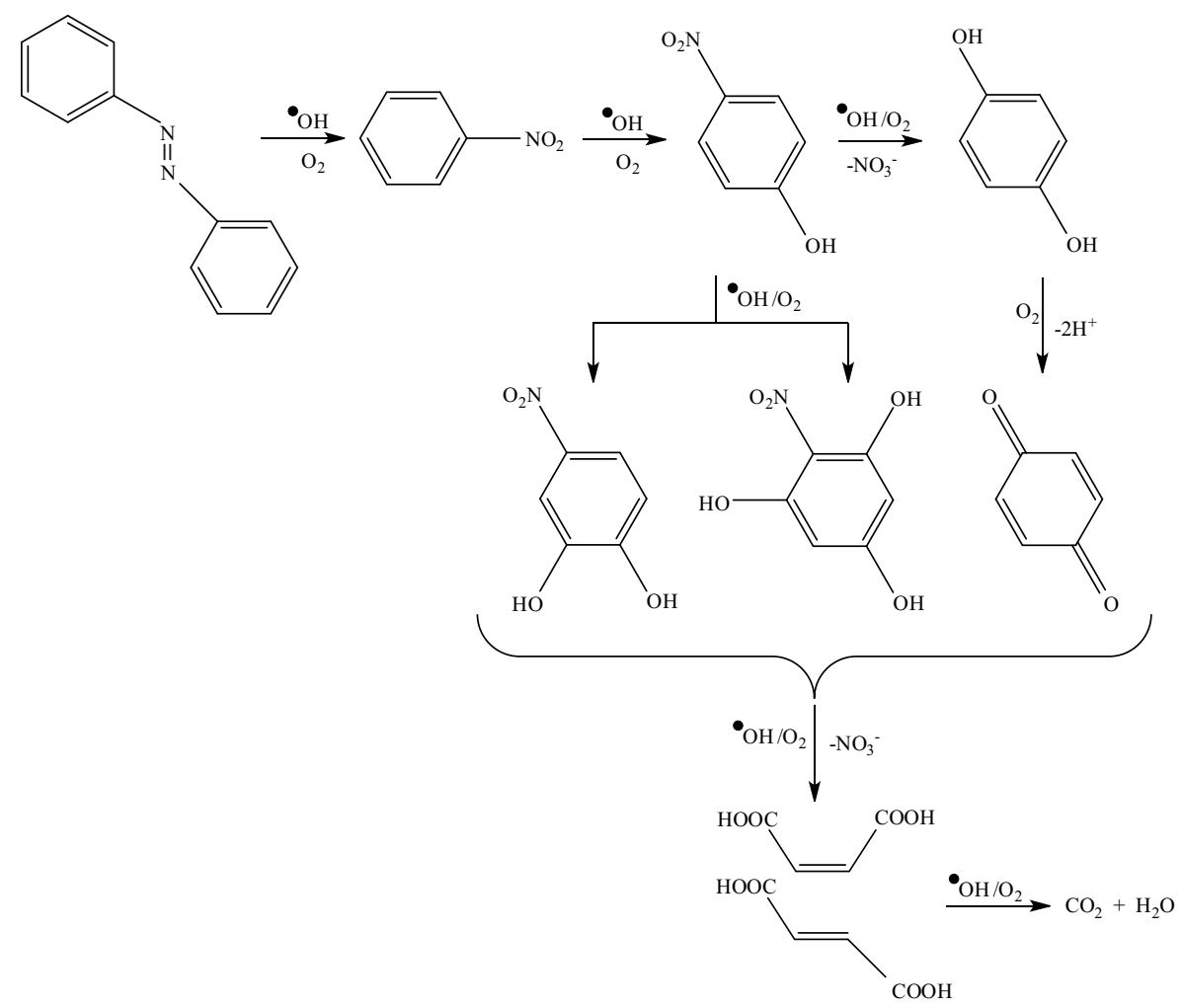

Figura 2. Posible mecanismo de reacción de la mineralización de Anaranjado de metilo por proceso Fenton

\section{PARTE EXPERIMENTAL}

Calibración de la técnica de espectrofotometría para la evaluación del avance de la decoloración del anaranjado de metilo (AM)

Se utilizó un espectrofotómetro Shimatzu UV/Vis previamente calibrado. En la figura 3 se observa el cambio en la longitud de onda debido a la modificación del pH del AM, observándose que en el rango de $\mathrm{pH}$ de 2,0 a 4,5 la longitud de onda se mantiene constante, aceptándose como apropiado el empleo de la técnica de espectrofotometría para el 
seguimiento de la decoloración debido a que pequeñas variaciones del $\mathrm{pH}$, producto de reacciones secundarias no afectarán la evaluación de la decoloración del AM.

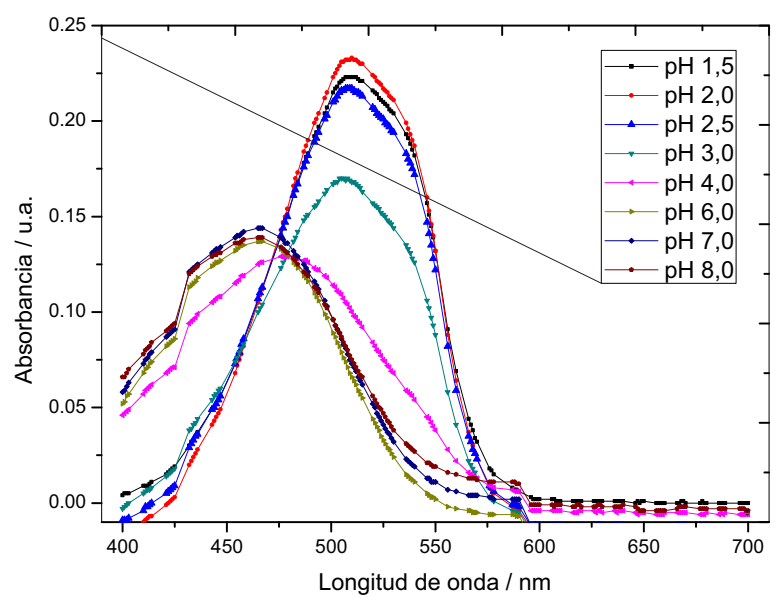

Figura 3: Barrido espectral de 2ppm de Naranjado de metilo, desde $400 \mathrm{~nm}$ a $700 \mathrm{~nm}$ variando el pH.

\section{Determinación del tiempo de decoloración óptimo variando los parámetros de cantidad de $\mathrm{H}_{2} \mathrm{O}_{2}, \mathrm{FeSO}_{4} \cdot 7 \mathrm{H}_{2} \mathrm{O}$ y concentración de $\mathrm{AM}$.}

Se realizó ensayos para la determinación del tiempo de decoloración variando los parámetros de concentración de $\mathrm{AM}, \mathrm{H}_{2} \mathrm{O}_{2}$ y $\mathrm{FeSO}_{4} .7 \mathrm{H}_{2} \mathrm{O}$, se obtuvo 89 puntos y los datos obtenidos fueron ingresados al programa Minitab obteniéndose el resultado mostrado en la figura 4 . De acuerdo al mapa de colores, que representa los rangos de tiempo, se observa que la zona con el menor tiempo de decoloración es el azul $<200$ segundos. Sin embargo, se observa que la relación de $\mathrm{H}_{2} \mathrm{O}_{2} / \mathrm{FeSO}_{4} \cdot 7 \mathrm{H}_{2} \mathrm{O}$ no es completamente lineal para todas las concentraciones por lo que se hace necesario establecer una relación óptima para una determinada concentración del componente orgánico.

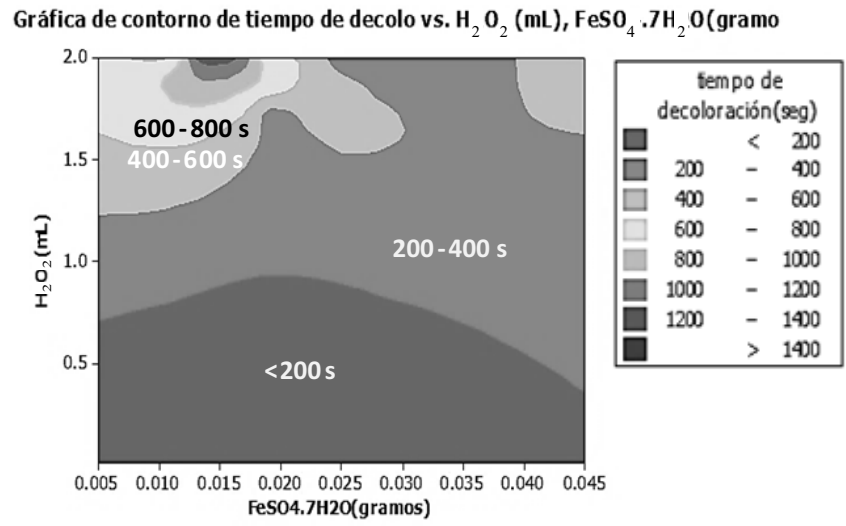

Figura 4: Gráfico en el software Minitab de optimización de parámetros, variando la cantidad de peróxido de hidrógeno, sulfato de hierro y anaranjado de metilo 


\section{Análisis del potencial de oxidación-reducción (ORP) de producción de oxidantes mediante el proceso Fenton.}

El análisis ORP se realizó utilizando un electrodo de platino y electrodo de referencia de $\mathrm{Ag} / \mathrm{AgCl}$, sumergiendo el electrodo en solución ORP estándar de $120 \mathrm{mV}$ antes y después de cada medida con previo enjuague en agua ultrapura de $18 \mathrm{M} \Omega$ de resistividad. La técnica ha mostrado su utilidad para estudios de la capacidad oxidante de sustancias en soluciones acuosas $^{8}$. En la figura 5 se presenta el gráfico correspondiente a la evaluación del potencial ORP, indicador de la máxima producción de oxidantes en la solución, frente al añadido de peróxido de hidrógeno determinándose el valor óptimo de $\mathrm{H}_{2} \mathrm{O}_{2}$ para $18 \mathrm{ppm}$ de sulfato de hierro, siendo ésta de $0,967 \mathrm{mM}$, aproximadamente, el cual corresponde a un valor aproximado $550 \mathrm{mV}$ de ORP; un alícuota adicional de $\mathrm{H}_{2} \mathrm{O}_{2}$, sobre el valor determinado, no contribuye a la mayor producción de oxidantes identificándose una relación óptima de $\mathrm{Fe}^{2+} / \mathrm{H}_{2} \mathrm{O}_{2}$ de 0,28 , similar a los obtenidos por Roger I. Méndez Novelo et al .

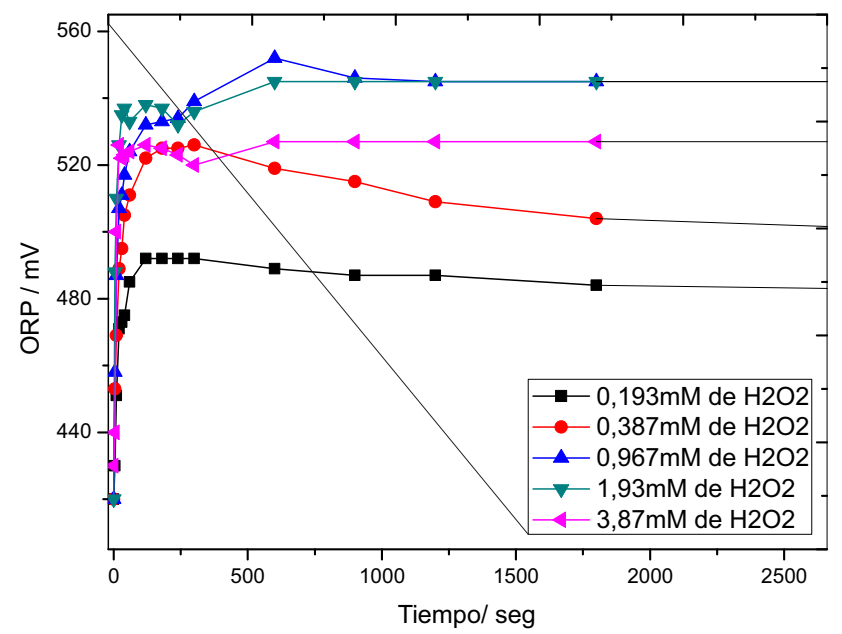

Figura 5: Valores de ORP en agua desionizada con $18 \mathrm{ppm}$ de sulfato de hierro variando la concentración de peróxido de hidrógeno

\section{Evaluación de la demanda química de oxígeno (DQO) de la muestra de AM tratado con el proceso Fenton.}

En la figura 6 se presenta el gráfico correspondiente al descenso del valor de DQO correspondiente al tratamiento Fenton de una solución de 100 ppm de Anaranjado de metilo, El valor del DQO en cada medición se realizó empleando el procedimiento de valoración empleando $\mathrm{Fe}\left(\mathrm{NH}_{4}\right)_{2}\left(\mathrm{SO}_{4}\right)$ a $0,1 \mathrm{~N}$ por retroceso del exceso de ion dicromato después de la reacción de oxidación de la muestra. En el gráfico se aprecia que la curva de descenso sigue una tendencia de tipo exponencial y asintótico a bajos valores de DQO. Este comportamiento es típico para los procesos Fenton debido a la interferencia del hierro que toma importancia a bajas concentraciones. Sin embargo la eficiencia del proceso Fenton es apreciable observándose que para 120 minutos de tratamiento se logra una reducción del DQO del $86,9 \%$. 


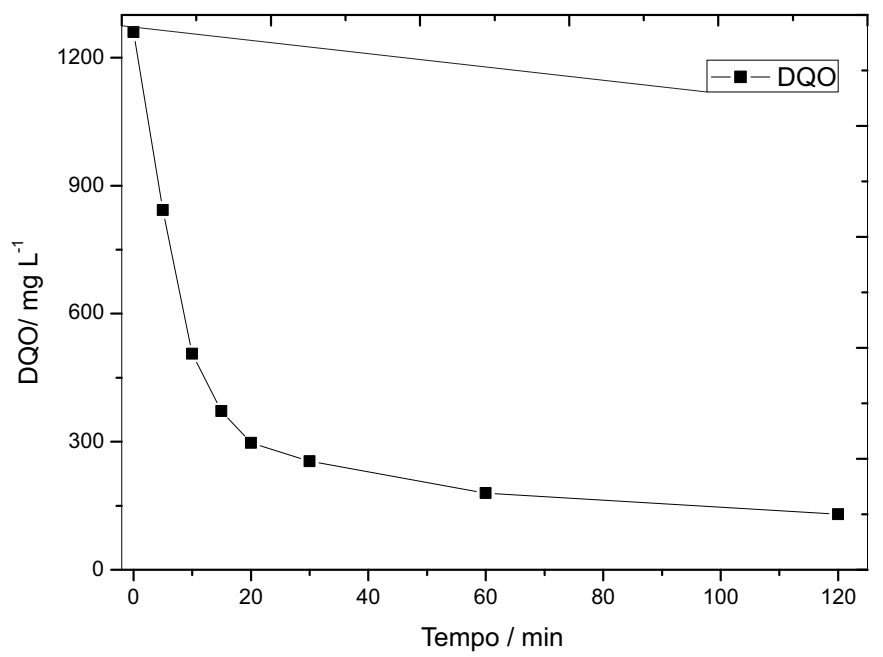

Figura 6: Gráfico del descenso del valor DQO correspondiente al anaranjado de metilo tratado con el proceso Fenton a una concentración de 18 ppm de sulfato de hierro y de $0,387 \mathrm{mM}$ de $\mathrm{H}_{2} \mathrm{O}_{2}$

\section{Análisis de la mineralización del contenido orgánico total (TOC) mediante el proceso Fenton}

Se realizó el tratamiento Fenton a una muestra de anaranjado de metilo de 100 ppm equivalente a $60 \mathrm{mg} / \mathrm{L}$ de contenido de carbono; se utilizó la misma proporción de sulfato de hierro y peróxido de hidrógeno establecido en el anterior ensayo. El análisis se realizó utilizando un equipo MICRO N/C Analityc Jena para el análisis de carbón orgánico total (TOC) el cual emplea un catalizador de micropartículas de platino y $680{ }^{\circ} \mathrm{C}$ de temperatura de combustión, se determina como $\mathrm{C}_{\text {total }}$ a partir del análisis de $\mathrm{CO}_{2}$. El protocolo de análisis establecido es tomar una alícuota de $200 \mu \mathrm{L}$ de muestra líquida la solución se inyecta a la cámara de combustión del equipo registrándose el resultado para cada medida. La figura 7 muestra la variación de los valores del TOC de la muestra de AM en función al tiempo de tratamiento. En el gráfico se observa que el decaimiento de los valores TOC a 120 minutos de tratamiento es de sólo 33,3\% con respecto al valor inicial, indicándonos el porcentaje de materia orgánica que pasa a $\mathrm{CO}_{2}$ de acuerdo a la Ec.6:

$$
\mathrm{AM}+\mathrm{O}_{2} \stackrel{\text { Fenton }}{\longrightarrow} \mathrm{CO}_{2}+\mathrm{H}_{2} \mathrm{O}+\mathrm{N}_{2}
$$

Es posible que el resto de la materia orgánica haya sufrido reacciones de oxidación, si bien no han conducido a su mineralización completa; puede haber derivado en moléculas orgánicas más simples, reflejado en el indicador del DQO, donde el grupo cromóforo necesariamente ha sido fraccionado como lo indica la decoloración mostrada en la figura 4. 


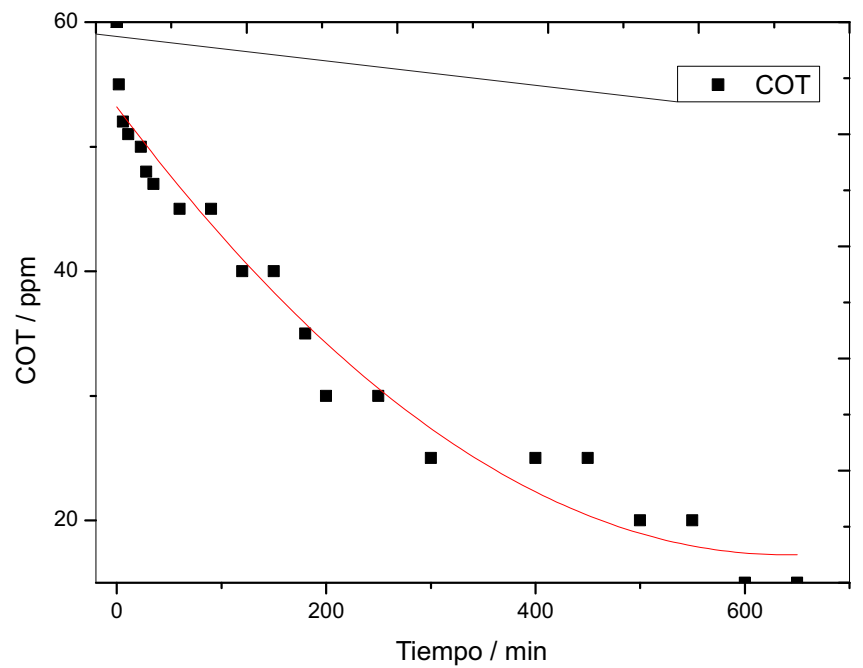

Figura 7: Gráfico de la variación del carbón orgánico total (TOC) de una solución de 100 ppm de AM en función al tiempo de tratamiento mediante Fenton; se utilizó una concentración de $18 \mathrm{ppm}$ de sulfato de hierro y de $0,387 \mathrm{mM}$ de $\mathrm{H}_{2} \mathrm{O}_{2}$

\section{Cálculo de las constantes de velocidad de reacción en la decoloración del anaranjado} de metilo (AM) mediante tratamiento Fenton

De acuerdo al proceso Fenton, la reacción más rápida y directa es la decoloración de la solución debido al rompimiento del grupo azoico el cual es de enlace simple al $\mathrm{pH}$ de tratamiento por lo tanto más susceptible a la reacción; sin embargo el proceso de oxidación no se detiene, continúa fraccionando las moléculas orgánicas a moléculas simples y parte de ellas mineralizándolas completamente convirtiéndolas en $\mathrm{CO}_{2}$ última etapa de la reacción. En esta sección se ha determinado la cinética de la reacción de decoloración y los datos fueron tomados mediante un espectrofotómetro

Se realizó la determinación de las constantes de velocidad y el orden de la reacción del proceso de decoloración para el cual se tomó una concentración inicial de $2 \mathrm{ppm}$ de $\mathrm{AM}$ a pH=2,5 y relación de concentración de $\mathrm{Fe}^{2+} / \mathrm{H}_{2} \mathrm{O}_{2}$ de 0,28 y 2 ppm de $\mathrm{Fe}^{2+}$

Para el cálculo se emplea la ecuación cinética:

$$
\operatorname{Ln}(-\mathrm{dC} / \mathrm{dt})=\mathrm{nLnC}+\mathrm{Lnk}
$$

Donde: $\mathrm{dC}$ es la diferencia de concentración en cada punto

$\mathrm{k}=$ es la constante de velocidad en unidades $\mathrm{s}^{-1}$

$\mathrm{n}=$ orden de reacción 
Tabla 1: Datos para la determinación de la constante de velocidad y orden de reacción en la decoloración de $200 \mathrm{~mL}$ de anaranjado de metilo 2 ppm mediante proceso Fenton

\begin{tabular}{|c|c|c|c|c|c|}
\hline $\begin{array}{c}\text { Tiempo } \\
(\text { segundos }\end{array}$ & $\begin{array}{c}\text { Concentración } \\
(\mathrm{ppm})\end{array}$ & $\mathrm{dC}$ & $\mathrm{dt}$ & $\mathrm{LnC}=\mathrm{x}$ & $\mathrm{Ln}(-\mathrm{dC} / \mathrm{dt})=\mathrm{y}$ \\
\hline 0 & 2 & & & & \\
\hline 2 & 1,96 & $-0,04$ & 2 & 0,673 & $-3,912$ \\
\hline 4 & 1,6 & $-0,36$ & 2 & 0,470 & $-1,715$ \\
\hline 6 & 1,4 & $-0,2$ & 2 & 0,336 & $-2,303$ \\
\hline 8 & 1,2 & $-0,2$ & 2 & 0,182 & $-2,303$ \\
\hline 12 & 1 & $-0,2$ & 4 & 0,000 & $-2,996$ \\
\hline 14 & 0,9 & $-0,1$ & 2 & $-0,105$ & $-2,996$ \\
\hline 20 & 0,8 & $-0,1$ & 6 & $-0,223$ & $-4,094$ \\
\hline 30 & 0,6 & $-0,2$ & 10 & $-0,511$ & $-3,912$ \\
\hline 42 & 0,4 & $-0,2$ & 12 & $-0,916$ & $-4,094$ \\
\hline 58 & 0,36 & $-0,04$ & 16 & $-1,022$ & $-5,991$ \\
\hline 64 & 0,34 & $-0,02$ & 6 & $-1,079$ & $-5,704$ \\
\hline 76 & 0,3 & $-0,04$ & 12 & $-1,204$ & $-5,704$ \\
\hline 92 & 0,26 & $-0,04$ & 16 & $-1,347$ & $-5,991$ \\
\hline 94 & 0,2 & $-0,06$ & 2 & $-1,609$ & $-3,507$ \\
\hline 98 & 0,14 & $-0,06$ & 4 & $-1,966$ & $-4,200$ \\
\hline 138 & 0,1 & $-0,04$ & 40 & $-2,303$ & $-6,908$ \\
\hline 162 & 0,06 & $-0,04$ & 24 & $-2,813$ & $-6,397$ \\
\hline 216 & 0,04 & $-0,02$ & 54 & $-3,219$ & $-7,901$ \\
\hline 250 & 0,02 & $-0,02$ & 34 & $-3,912$ & $-7,438$ \\
\hline & & & & \\
\hline
\end{tabular}

A partir de los valores generados, mostrados en la tabla 1, se hace una regresión lineal sobre los valores de $\mathrm{X}$ e $\mathrm{Y}$; se construye el gráfico de la figura 8 y se obtiene los valores de " $\mathrm{k}$ ” y " $\mathrm{n}$ " (Ec.7). 


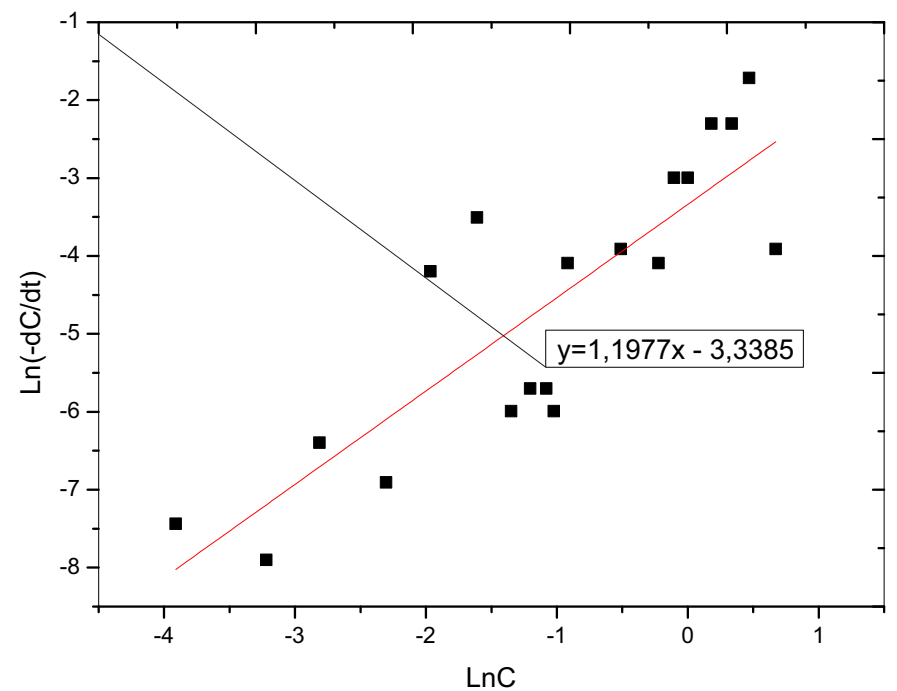

Figura 8: Gráfica para la determinación de la constante de velocidad y orden de reacción correspondiente a la decoloración de $200 \mathrm{~mL}$ de solución con $2 \mathrm{ppm}$ de anaranjado de metilo.

La ecuación de la recta del gráfico es:

$\mathrm{Ln}(-\mathrm{dC} / \mathrm{dt})=1,1977 \mathrm{LnC}-3,3385$

Luego $\mathrm{k}=0,035 . \mathrm{s}^{-1}$

$\mathrm{n}=1,19$ equivalente $\mathrm{a} n=1$

Cinética de la reacción de oxidación evaluada mediante la demanda química de oxígeno (DQO)

Se utiliza los datos de la figura 6, en términos de $\operatorname{Ln}(-\mathrm{d}(\mathrm{DQO}) / \mathrm{dt})$ vs. Ln(DQO)

Empleando la ecuación cinética:

$\operatorname{Ln}(-\mathrm{d}(\mathrm{DQO}) / \mathrm{dt})=\mathrm{nLn}(\mathrm{DQO})-\mathrm{Lnk}$

En la figura 9 se muestra la relación lineal, siendo la ecuación representativa:

$\operatorname{Ln}(-d(D Q O) / d t)=2,6649 \operatorname{Ln}(\mathrm{DQO})-12,893$ 


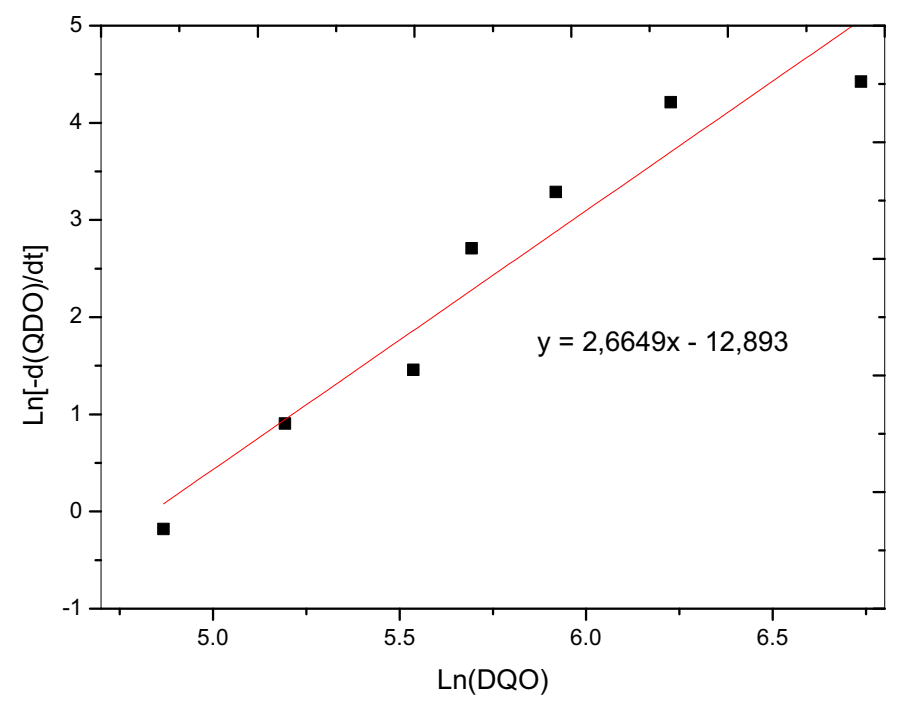

Figura 9: Gráfica para la determinación de la constante de velocidad y orden de reacción evaluado mediante la DQO de $200 \mathrm{~mL}$ de solución con 2 ppm de anaranjado de metilo

Luego $\mathrm{k}=0,0000025 \mathrm{~s}^{-1}$

$\mathrm{n}=2,66$ equivalente $\mathrm{n}=2$

\section{CONCLUSIONES}

- $\quad$ El proceso Fenton permite tratar efluentes con alto contenido orgánico, el cual puede ser aplicado para la mineralización de la materia orgánica, disminución del DQO y decoloración de soluciones que contienen compuestos azoicos, como es el caso de las aguas de desecho del teñido en la industria textil.

- La relación óptima de $\mathrm{Fe}^{2+} / \mathrm{H}_{2} \mathrm{O}_{2}$ para el tratamiento del compuesto azoico anaranjado de metilo fue de 0,28

- La constante de velocidad para la decoloración del AM fue de $\mathrm{k}=0,035 . \mathrm{s}^{-1} \mathrm{y}$ el orden de reacción de $n=1$

- $\quad$ La constante de velocidad para la disminución de la DQO fue de $\mathrm{k}=0,0000025 \mathrm{~s}^{-1} \mathrm{y}$ orden de reacción de $n=2$, mostrando consistencia con el modelo de múltiples reacciones de fraccionamiento de las moléculas orgánicas del proceso Fenton

\section{AGRADECIMIENTOS}

Al Instituto de investigación de la Universidad Nacional de Ingeniería (IGI) por los fondos destinados a la presente investigación. 


\section{BIBLIOGRAFÍA}

1. Carlos A. Martínez-Huitle, Enric Brillas, «Decontamination of wastewaters containing synthetic organic dyes by electrochemical methods: A general review,» Applied Catalysis B: Environmental, 2009; 87: 105-145,

2. Enric Brillas, Ignasi Sirés, Mehmet A. Oturan, «Electro-Fenton Process and Related Electrochemical Technologies Based on Fenton's Reaction Chemistry,» Chemical Reviews, 2009; 109; 6570-6631

3. Barb, W.G.; Baxendale, J.H.; George, P.; Hargrave, K.R. Reactions of ferrous and ferric ions with hydrogen peroxide Nature, 1949; 163: 692-694

4. Jiju M. Joseph, Hugo Destaillats, Hui-Ming Hung, and Michael R. Hoffmann, «The Sonochemical Degradation of Azobenzene and Related Azo Dyes: Rate Enhancements via Fenton's Reactions,» The Journal of Physical Chemistry A, 2000; 104: 301-307

5. Kavitha y Palanivelu, «The role of ferrous ion in Fenton and photo-Fenton processes for the d degradation of phenol,» Chemosphere, 2004; 55: 1235-1243,

6. Maciel, Sant'Anna, Dezotti, «Phenol removal from high salinity effluents using Fenton's reagent and photo-Fenton reactions,» Chemosphere, 2004; 57: 711-719

7. Elodie Guivarch, Stephane Trevin, Claude Lahitte, Mehmet A. Oturan, «Degradation of azo dyes in water by Electro-Fenton process» Environmental Chemistry Letters, 2003; 1: $38-44$

8. Pilar del Socorro Lázaro Rubio, Adolfo La Rosa-Toro Gómez, Evaluación de electrodos basados en $\mathrm{SnO}_{2}$ dopados con $\mathrm{Sb}$, Ru y $\mathrm{TiO}_{2}$, fabricados por la técnica Pechini, Rev Soc Quím Perú 2013; 79 (2): 150-161

9. Roger I. Méndez N., José A. Pietrogiovanna B., Beatriz Santos O., María R. Sauri R., Germán Giácoman V., Elba R. Castillo B., «Determinación de la dosis óptima de reactivo Fenton en un tratamiento de lixiviados por Fenton-adsorción» Revista internacional de contaminación ambiental, 2010;26(3):211-220 